\title{
Europäische Zusammenarbeit in der Bewertung von Medizinprodukten - ein Schritt zur grenzüberschreitenden Gesundheitsversorgung
}

\author{
European Collaboration in Medical Device Assessment: One Step
} Towards Cross-Border Health Care

(ㄷ) (i) (ㅇ) $\odot$

\section{Autoren}

Julia Mayer, Sabine Ettinger, Anna Nachtnebel

Institute

Ludwig Boltzmann Institut für Health Technology Assessment, Wien, Österreich

\section{Schlüsselwörter}

Health Technology Assessment, Medizinprodukte, grenzüberschreitende Gesundheitsversorgung, Europäische Zusammenarbeit, EUnetHTA

Key words

health technology assessment, medical devices, cross-border health care, European collaboration, EUnetHTA

\section{Bibliografie}

DOI https://doi.org/10.1055/s-0043-109859

Online-Publikation: 19.6.2017

Gesundheitswesen 2018; 80: 210-216

(c) Georg Thieme Verlag KG Stuttgart · New York ISSN 0941-3790

\author{
Korrespondenzadresse \\ Julia Mayer \\ Ludwig Boltzmann Institut für \\ Health Technology Assessment \\ Garnisongasse 7/20 \\ 1090 Wien \\ Österreich \\ julia.mayer@hta.lbg.ac.at
}

\section{ZUSAMMENFASSUNG}

Ziel Ziel des Artikels ist die Darstellung rechtlicher Rahmenbedingungen sowie Erfahrungen in der grenzüberschreitenden Zusammenarbeit bei der Bewertung von Medizinprodukten als Grundlage für Erstattungsentscheidungen. Der mögliche Nutzen transnationaler Kooperation für verschiedene Stakeholdergruppen soll erläutert werden.

Methodik Prozesse und Herausforderungen der abgeschlossenen European Network for Health Technology Assessment (EUnetHTA) Joint Action 2 (JA2) werden zusammengefasst und diskutiert. Die dem Artikel zugrunde liegenden Erfahrungen beruhen auf der Erstellung und Evaluierung von insgesamt 6
Rapid Assessments zu Medizinprodukten, welche während der EUnetHTA JA2 in internationaler Kollaboration verfasst und publiziert wurden. Nutzen, Ziele und Chancen der laufenden EUnetHTA Joint Action 3 (JA3) werden dargestellt.

Ergebnisse Herausforderungen in der europäischen Medizinproduktebewertung umfassen die Themenauswahl und den Zeitpunkt der Bewertung, die Intransparenz des Medizinproduktemarkts sowie das Fehlen europäischer Standards zum systematischen Einbeziehen von PatientInnen. Charakteristika wie inkrementelle Veränderungen und Lernkurven machen es notwendig, Medizinprodukte über ihren gesamten Lebenszyklus hinweg zu begleiten. Der Nutzen der europäischen Zusammenarbeit für Stakeholder ist vielfältig: die aufgrund fehlender Evidenz herrschende Unsicherheit zum Zusatznutzen einer Technologie kann durch Early Dialogues reduziert werden; ein europaweit harmonisierter, transparenter Bewertungsprozess steigert die Nachvollziehbarkeit und Qualität der Berichte; die Arbeitsteilung unter den Health Technology Assessment (HTA)Organisationen ermöglicht eine ressourcenschonende Bewertung einer größeren Anzahl an Technologien; die Patientenbeteiligung gewährleistet die Berücksichtigung patientenrelevanter Endpunkte. Die Wichtigkeit der grenzübergreifenden Zusammenarbeit im Bereich HTA zeigt sich in der Weiterführung des EUnetHTA Projekts, welches die Festigung der internationalen Kollaboration auch nach Auslaufen der EU-Förderungen zum Ziel hat.

Schlussfolgerung Eine nachhaltige transnationale Kooperation in der Bewertung von Medizintechnologien kann die grenzüberschreitende Gesundheitsversorgung und die effiziente Zusammenarbeit der nationalen Gesundheitssysteme sichern. Der Fokus sollte dabei auf einer flächendeckenden Implementierung gemeinsam entwickelter Methoden und Qualitätsstandards liegen. Die europäische Zusammenarbeit kann zu einem konkreten Nutzen für PatientInnen, ÄrztInnen, HTA-Organisationen und nationale EntscheidungsträgerInnen führen.

\section{ABSTRACT}

Aim The aim of this paper was to present the legal framework as well as previous experience in transnational collaboration regarding the assessment of medical devices for reimburse- 
ment decisions. Furthermore, the possible benefit of transnational collaboration for various stakeholders will be discussed.

Methods Experiences gathered with the compilation of overall 6 joint rapid assessments of medical devices within the completed European Network for Health Technology Assessment (EUnetHTA) Joint Action 2 (JA2) are summarised and discussed. Benefits, aims and opportunities of the ongoing EUnetHTA Joint Action 3 (JA3) are described.

Results Challenges in joint European assessment of medical devices encompass the choice of topics and the time point of assessments, the non-transparency of the medical devices market as well as the lack of European standards for systematic patient involvement. Characteristics of medical devices such as incremental changes and learning curves call for monitoring of medical devices through their whole lifecycle. The concrete benefit of European collaboration for stakeholders is manifold: uncertainties with regard to the actual added value of a technology, caused by a lack of evidence, may be reduced by Early Dialogues; harmonized and transparent assessment processes throughout Europe increase the reproducibility and quali- ty of reports; the division of work among the health technology assessment (HTA) organisations allows a resource efficient assessment of a larger amount of technologies; patient involvement facilitates consideration of patient-relevant endpoints. The importance of crossborder collaboration in the field of HTA is shown in the continuation of the EUnetHTA project, which aims to further strengthen international collaboration even after expiration of EU funding.

Conclusion A sustainable transnational collaboration in the assessment of medical devices can ensure cross-border health care as well as efficient collaboration of national health systems. The focus should be on a wide implementation of jointly established methods and quality standards. The European collaboration can lead to a concrete benefit for patients, physicians, HTA organisations and national decision makers.

\section{Einleitung}

Auch in der Gesundheitsversorgung kommt es zunehmend zur Globalisierung: neue Medizinprodukte werden international vermarktet, und sowohl PatientInnen als auch die Öffentlichkeit erwarten - in Anbetracht der zunehmenden Mobilität der Bevölkerung - eine einheitlich gute Gesundheitsversorgung in Europa. Durch die Direktive 2011/24/EU zur grenzüberschreitenden Gesundheitsversorgung [1] wurde dieser Entwicklung und damit auch der Erwartungshaltung von PatientInnen, abseits ihrer Herkunftsländer medizinische Versorgung höchster Qualität in Anspruch nehmen zu können [2], Rechnung getragen.

Gleichzeitig stoßen die Mitgliedsstaaten durch steigende Gesundheitsausgaben zunehmend an ihre finanziellen Grenzen. Um dennoch eine optimale Gesundheitsversorgung mit sicheren und wirksamen Technologien gewährleisten zu können, haben in den letzten Jahrzehnten viele Länder Health Technology Assessment (HTA)-Institute gegründet, die neue Technologien vor der Aufnahme in nationale Leistungskataloge anhand wissenschaftlicher Kriterien evaluieren und so evidenzbasierte Kostenerstattungsentscheidungen ermöglichen.

Da Kostenerstattungsentscheidungen aber auf nationaler oder regionaler Ebene getroffen werden, entstehen europaweit Redundanzen in der Bewertung neuer Technologien, und limitierte finanzielle und personelle Ressourcen werden gebunden. Mit der Direktive 2011/24/EU wurde aber auch der Grundstein für die Zusammenarbeit im Bereich HTA gelegt. Durch die darin vorgesehene europaweite Zusammenarbeit soll eine effizientere Arbeitsweise und gleichzeitig eine bestmögliche, europaweit standardisierte Patientenversorgung verwirklicht werden.

Das EU-Projekt EUnetHTA (European Network for Health Technology Assessment) verfolgt bereits seit 2006 das Ziel, HTA-Ressourcen effizienter zu nutzen und die transnationale Zusammenarbeit sowie den Informationsaustausch nachhaltig zu fördern [3]. Beginnend mit einem EU-Projekt (2006-2008) und 2 weiteren Joint
Actions (JA 2010-2012 und JA2 2012-2015) wurden Prozesse und Instrumente entwickelt, um Redundanzen in der EU-weiten HTAProduktion zu vermeiden. In der JA3 (2016-2020) sollen bestehende Methoden und Prozesse weiter verbessert und ergänzt werden.

Im Gegensatz zu Medikamenten, deren Bewertung vor Kostenerstattungsentscheidungen in den meisten europäischen Ländern bereits Routine ist, sind HTAs zu Medizinprodukten weit weniger einheitlich und systematisiert, obwohl auch Medizinprodukte maßgeblich an steigenden Gesundheitsausgaben beteiligt sind. Ziel dieses Artikels ist daher, rechtliche Rahmenbedingungen darzustellen, welche die Bewertungen von Medizinprodukten erschweren, sowie Erfahrungen, die bei der internationalen Bewertung von Medizinprodukten im Rahmen von EUnetHTA gesammelt wurden, mit einem breiteren Publikum zu teilen; der mögliche Nutzen des Projekts soll abseits der rein akademisch-wissenschaftlichen Komponente auch für andere Stakeholder wie ÄrztInnen und PatientInnen erläutert werden.

\section{Methodik}

Prozesse und Herausforderungen der 2015 abgeschlossenen EUnetHTA JA2, die insbesondere mit der Bewertung von Medizinprodukten vergesellschaftet sind, werden zusammengefasst und diskutiert. Weiters wird der mögliche Nutzen für PatientInnen und ÄrztInnen, der sich aus gemeinsamen HTAs zu nicht-medikamentösen Technologien ergibt, dargestellt. Abschließend wird ein Ausblick auf Ziele und Chancen der Zusammenarbeit in der EUnetHTA JA3 gegeben.

Die dem Artikel zugrunde liegenden Erfahrungen beruhen auf der Erstellung und Evaluierung von insgesamt 6 Rapid Assessments zu Medizinprodukten, welche während der EUnetHTA JA2 in internationaler Kollaboration verfasst und publiziert wurden [4]. Zwischen 5 und 9 europäische HTA-Institute waren bei der Erstellung der einzelnen Rapid Assessments als Autorlnnen und Reviewer involviert; der gesamte Prozess wurde durch ein Koordinationsteam abgestimmt. 
Stakeholder wurden auf unterschiedliche Weise involviert ( $\triangleright$ Abb. 1): die Autorlnnen wurden jeweils von 1 bis 3 klinischen ExpertInnen beraten, um die Auswahl von relevanten Vergleichsprodukten, aber auch, um die Richtigkeit klinischer Inhalte der Assessments zu gewährleisten. Die Produkt-Hersteller wurden zu Beginn des Assessment-Prozesses kontaktiert, um bspw. die Identifikation weiterer Hersteller zu erleichtern, und ab dem fünften Assessment auch, um Scoping Meetings zu planen und einen Dossier-basierten Informationsaustausch einzuleiten. Insgesamt wurden 25 Produkt-Hersteller kontaktiert und in den Assessment-Prozess involviert; an den 2 Scoping-Meetings nahmen in Summe 6 Hersteller (von 13) teil, während Dossiers von insgesamt 9 Herstellern (von 13) eingereicht wurden. PatientenvertreterInnen wurden einbezogen, um die Patientenrelevanz der gewählten Endpunkte und die Angemessenheit der Schlussfolgerungen aus Patientensicht zu gewährleisten; insgesamt unterstützten 4 Patientenorganisationen die Produktion von 3 Assessments mit Kommentaren und Feedback.

\section{Ergebnisse}

\section{Gemeinsame Medizinproduktebewertung in der EUnetHTA JA2}

Die während der EUnetHTA JA2 durch die oben beschriebenen Prozesse erstellten englischsprachigen Berichte enthalten eine Beschreibung der Technologie und der Indikation sowie eine systematische Bewertung von Wirksamkeit und Sicherheit, und schließen mit einer Interpretation der verfügbaren Evidenz.

Gemäß des „Assess global, decide local“-Gedankens von EUnetHTA enthalten diese gemeinsam verfassten Rapid Assessments keine Empfehlungen; diese sollen auf nationaler oder regionaler
Ebene ergänzt werden und damit unter Berücksichtigung von Kontext-spezifischen Faktoren an die jeweiligen Gesundheitssysteme angepasste Kostenerstattungsentscheidungen ermöglichen.

Die Themen der Assessments wurden in erster Instanz aus den nationalen Arbeitsprogrammen der Erstautorlnnen gewählt. Als Basiskriterium für die Themenauswahl wurde zunächst das Bestehen einer CE-Zertifizierung definiert, in weiterer Folge wurden aber Priorisierungen durch die teilnehmenden HTA-Institutionen unternommen, um die Relevanz für möglichst viele Länder zu gewährleisten.

Basierend auf diesen EUnetHTA Rapid Assessments zu Medizinprodukten wurden bislang (Status: Sommer 2016) 11 nationale Berichte veröffentlicht [5].

\section{Rechtliche Rahmenbedingungen und Herausforderun- gen in der europäischen Medizinproduktebewertung}

Themenauswahl und Zeitpunkt der Bewertung Durch das Fehlen einheitlicher Prozesse zur Markteinführung von Medizinprodukten variieren - im Gegensatz zu Medikamenten - sowohl die Zeitpunkte der nationalen Antragstellung als auch anstehende Kostenerstattungsentscheidungen. Nachdem aber nur jene Rapid Assessments für nationale Entscheidungen Verwendung finden, die sowohl zeitlich als auch thematisch nationale Fragestellungen abdecken, sind die Relevanz der gewählten Themen und auch das Timing die wichtigsten Faktoren für die Wiederverwendung der europäischen Assessments zur Erstellung nationaler Berichte.

In der EU basieren die Zulassung und der Marktzugang von Medizinprodukten derzeit noch auf 3 Richtlinien [6-8]. Ziel ist der höchstmögliche Schutz der Gesundheit und Sicherheit der NutzerInnen und ein gut funktionierender EU-Binnenmarkt $[9,10]$. Alle Medizinprodukte, die auf den europäischen Markt kommen, müssen mit der CE-Kennzeichnung versehen sein, die ihre Übereinstimmung mit den Vorschriften bescheinigt [10]. Für die Konformitäts-

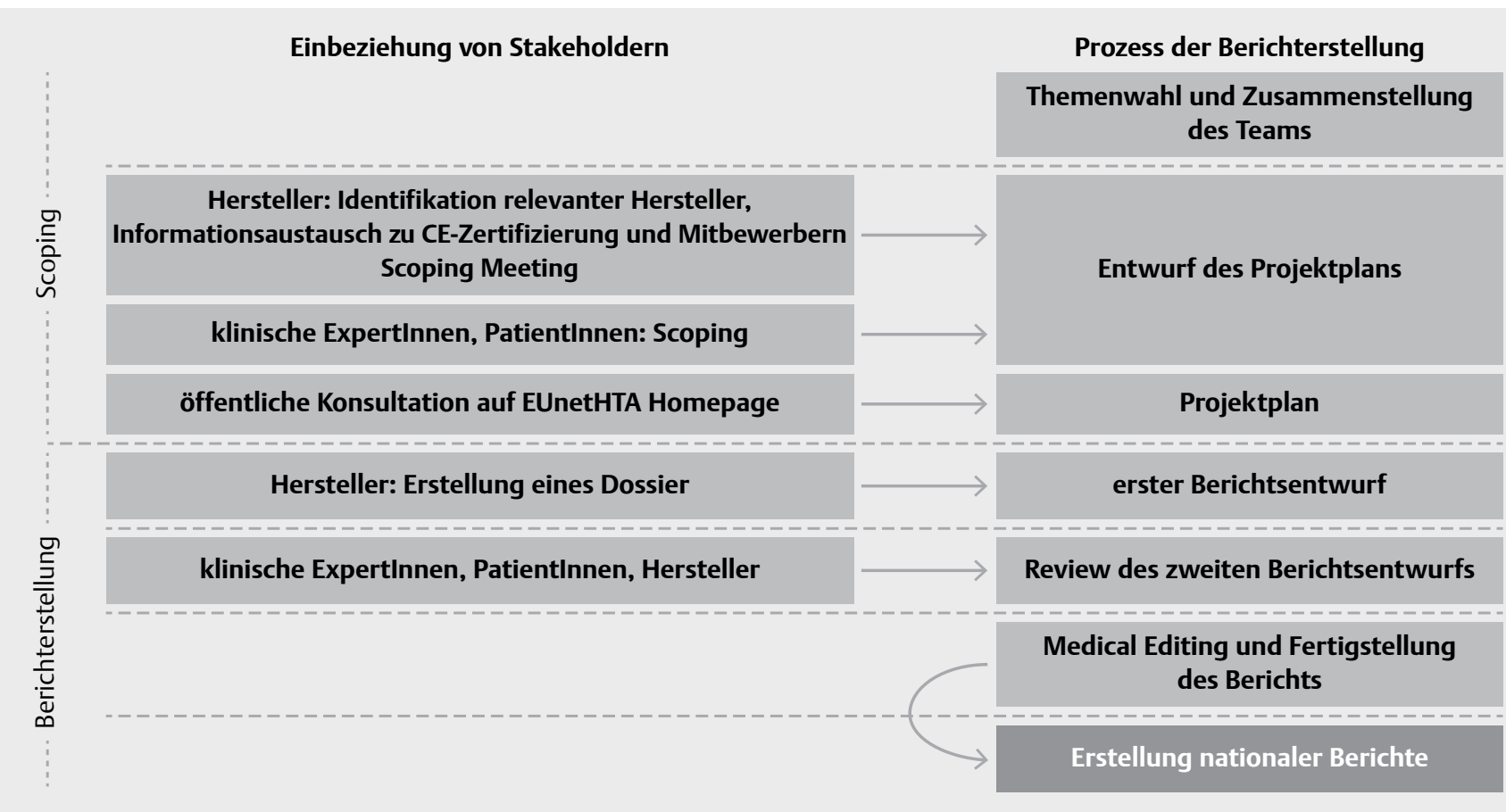

Abb. 1 Einbeziehung von Stakeholdern in den Assessment-Prozess in der EUnetHTA JA2. 
- Tab. 1 Zulassung von Medizinprodukten in der EU $[7,10]$.

\begin{tabular}{|l|l|l|l|}
\hline Verantwortliche Stelle (Instrument) & \multicolumn{1}{|l|}{ Benannte Stellen - dezentralisiertes System profitorientierter Organisationen (CE Zertifizierung) } \\
\hline & Risikoklasse I & Risikoklasse Ila & Risikoklasse Ilb \\
\hline Prozess & Selbstzertifizierung (nicht sterile & Konformitätsbewertung durch Benannte Stelle \\
& Produkte ohne Messfunktion) & \\
\hline Anforderungen für die Zulassung & 1. Grundlegende Anforderungen \\
& 2. Allgemeine Anforderungen: Sicherheit, Leistung, Risiken \\
& - Anforderungen an die Auslegung und Konstruktion (Auszug) \\
& - Nebenwirkungen \\
& - Chemische, physikalische und biologische Eigenschaften \\
& - Infektion und mikrobielle Kontamination \\
& - Schutz vor Strahlungen \\
\hline Evidenzanforderungen & (kleine) Klinische Studien, Labortests, Literaturübersichten \\
\hline Transparenz der Entscheidung & Keine veröffentlichten Unterlagen \\
\hline
\end{tabular}

bewertung von Mittel- und Hochrisikotechnologien ist eine Benannte Stelle (z. B. eine nationale Normungsbehörde) zuständig, die das Produkt hinsichtlich der Sicherheits- und Leistungsstandards überprüft. Aktuell sind 59 Benannte Stellen [11] unter den 3 relevanten Richtlinien [6-8] gemeldet. Im Gegensatz zur harmonisierten Arzneimittelzulassung, die einheitlich für Europa durch die European Medicines Agency erfolgt, ist das System für Medizinprodukte dadurch stark fragmentiert. $>$ Tab. 1 fasst die Charakteristika der Medizinproduktezulassung in Europa zusammen.

Neben der gemeinsamen Auswahl der Themen, ist auch das Timing der gemeinsamen Assessments ein wichtiges Kriterium. Da im Rahmen der Konformitätsbewertung die klinische Wirksamkeit und der tatsächliche Nutzen für PatientInnen derzeit nicht untersucht werden müssen, ist die Evidenzbasis zum Zeitpunkt der Zulassung von Medizinprodukten sehr eingeschränkt; bei sehr frühen Assessments sind kontrollierte Studien, insbesondere randomisiert kontrollierte Studien (RCTs), die den Goldstandard für die Einschätzung der Wirksamkeit im Vergleich zu therapeutischen Alternativen darstellen, im Gegensatz zu Arzneimitteln daher nur selten verfügbar [12,13]. Schlussfolgerungen und Empfehlungen sind bei diesen früheren Assessments oft mit großen Unsicherheiten vergesellschaftet [12]. Dementsprechend wurden am Anfang der JA2 eher frühe Assessments durchgeführt. Nachdem aber zunehmend fraglich wurde, ob frühe Assessments mit geringer Datenlage einen optimalen Ressourcenverbrauch im Rahmen der internationalen Zusammenarbeit darstellen, verlagerte sich der Fokus zunehmend auf spätere Assessments zu Technologien mit komparativer Evidenz.

Demnächst soll die rechtliche Grundlage durch 2 neue Medizinprodukteverordnungen aktualisiert werden, durch die die Kontrolle bzw. Überwachung der Produkte vor und nach Markteinführung verschärft wird $[13,14]$. Neuerungen sind u. a. die Forderung nach stringenterer klinischer Evidenz, eine rigorosere Überwachung nach Markteinführung und nachträgliche Kontrollen der Bewertung von Hochrisikoprodukten [13, 15]. Voraussichtlich werden beide Verordnungen Ende 2016 erlassen und 3 bzw. 5 Jahre nach ihrer Veröffentlichung zur Anwendung kommen [13]. Es bleibt zu hoffen, dass die Entscheidungsgrundlage kurz nach Marktzulassung dadurch zumindest verbessert wird; eine 2016 in Deutschland durchgeführte Studie zeigte, dass sowohl Kostenträger als auch Anwender - insbesondere bei Hochrisikoprodukten - bereits zum Zeitpunkt der Konformitätsprüfung einen klaren evidenzbasierten Nachweis von Wirksamkeit und Patientennutzen wünschen. Auch die Veröffentlichung der Studienergebnisse und die damit gesteigerte Transparenz werden gefordert [16]. Eine ähnlich reglementierte Zulassung wie bei Arzneiprodukten, die meist große RCTs erfordert, ist aber nicht zu erwarten.

Intransparenter Medizinproduktemarkt Anders als der Arzneimittelmarkt ist der Medizinproduktemarkt durch eine Vielzahl an kleinen und mittleren Unternehmen stark fragmentiert. Eine Technologie wird oftmals von vielen Herstellern angeboten, wobei sich die Produkte rasch weiterentwickeln. Inkrementelle Entwicklungen mit oftmals nur geringen Unterschieden und kurze Lebenszyklen der Produkte [12] führen zu einem Angebot ähnlicher Technologien, die sich allerdings in verschiedenen Stadien ihres Produktlebenszyklus befinden und sich daher oft nur marginal unterscheiden. Die Identifikation aller relevanten Produkte für ein Assessment wird dadurch höchst komplex und zeitaufwändig, was v. a. für kleine HTA-Institute mit begrenzten Ressourcen eine Herausforderung darstellt. Eine mögliche Lösung wäre die Erstellung von Single Technology Assessments, die lediglich ein Produkt eines einzelnen Herstellers bewerten; im Rahmen von EUnetHTA wurden in der Regel aber die umfassenden Multi Technology Assessments vorgezogen, da sie die Relevanz des Assessments für den gesamten europäischen Markt erhöhen.

Beteiligung von PatientInnen und ExpertInnen Obwohl international Einigkeit darüber herrscht, dass die Einbeziehung von PatientInnen in HTA-Prozesse wertvolle Informationen liefern kann und einige Länder bereits Prozesse implementiert haben, fehlen bislang europäische Standards [17]. Insbesondere im nationalen Kontext verfügen (kleine) HTA-Institute oft nicht über die nötigen zeitlichen Ressourcen, um effiziente Methoden zu implementieren. In europäischen Assessments ist aber die Patientenbeteiligung ein wichtiges Thema: in der JA2 lag der Fokus auf der Pilotierung der Zusammenarbeit der HTA-Institute, standardisierte Prozesse zur Patientenbeteiligung waren noch nicht vorhanden. PatientInnen und Expertlnnen wurden in Assessments miteinbezogen, jedoch war dies nur eingeschränkt erfolgreich: nicht für alle Themen konnten geeignete PatientenrepräsentantInnen identifiziert und zur Mitwirkung motiviert werden, obgleich neben einzelnen Organisationen auch Dachorganisationen innerhalb EUnetHTAs kontaktiert wurden. Obwohl auch versucht wurde, europäische medizini- 
sche Fachgesellschaften mit einzubeziehen, um supranational gültige, klinische Vorgehensweisen abzubilden, gestaltete sich dies ebenso ressourcenintensiv und schwierig: ein Großteil der kontaktierten Gesellschaften reagierte entweder nicht oder abschlägig auf Anfragen. Erfolgreicher war die direkte Kontaktaufnahme mit einzelnen Expertlnnen.

\section{Begleitung des gesamten Lebenszyklus}

Bedingt durch die Besonderheiten von Medizinprodukten - fehlende komparative Studien bei Marktzulassung, inkrementelle Veränderungen während des Produktlebenszyklus sowie die Lernkurve bei den AnwenderInnen - ist es notwendig, diese über ihren gesamten Lebenszyklus hinweg zu begleiten: von Early Dialogues zwischen HTA-Institutionen, Herstellern und anderen Stakeholdern (bspw. nationalen EntscheidungsträgerInnen) in der Phase vor der Zulassung über frühe Assessments (Rapid Assessments) kurz nach Marktzulassung bis zu umfassenden Assessments bereits etablierter Technologien (Comprehensive Assessments).

Early Dialogues haben zum Ziel, unterschiedliche Anforderungen der für den Marktzugang verantwortlichen Akteure bezüglich Studiendesign, -qualität oder der Auswahl von Endpunkten, aufeinander abzustimmen; nachdem die Durchführung von RCTs in großen Patientenpopulationen kostspielig ist, soll durch die Einbindung von Zulassungsbehörden, HTA-Institutionen und Zahlern gewährleistet werden, dass die für Zulassungszwecke generierte Evidenz auch für Kostenerstattungsentscheidungen relevante Fragestellung beantworten kann. Während der JA2 wurden neben Arzneimitteln erstmals auch Medizinprodukte Gegenstand dieses Austausches.

Ist ein Medizinprodukt bereits am Markt etabliert, steigt die Verfügbarkeit (kontrollierter) Studien, die Evidenzbasis wird größer und robuster. Unsicherheiten bezüglich der Wirksamkeit und Sicherheit in der klinischen Praxis werden reduziert und etwaige Subgruppen, die besonders von der Technologie profitieren können, kristallisieren sich heraus. Um dieser Entwicklung Rechnung zu tragen, ist die Wiederbewertung von Medizinprodukten angezeigt. Zunächst nur bedingte Kostenerstattungsentscheidungen, die z. B. an die Generierung weiterer Studien geknüpft sind, können durch eine erneute Bewertung der Medizinprodukte in endgültige Entscheidungen umgewandelt werden. Umfassende Assessments, die neben reinen Wirksamkeits- und Sicherheitsaspekten auch ethische oder organisatorische Fragen behandeln, sind zwar zeitintensiver, bieten aber im Vergleich zu den schlankeren Rapid Assessments ein breiteres Bild aller relevanten Aspekte und können, etwa als Re-Assessment, die Information früher Berichte ergänzen.

\section{Diskussion}

\section{Nutzen für PatientInnen und ÄrztInnen}

Neben den Vorteilen für HTA-Organisationen und EntscheidungsträgerInnen im Gesundheitssystem, ergibt sich aus der europäischen Zusammenarbeit insbesondere für PatientInnen und Ärztlnnen ein konkreter Nutzen:

- Die aufgrund limitierter Evidenz herrschende Unsicherheit zum tatsächlichen Zusatznutzen von Medizinprodukten kann - wenn gemeinsam erarbeitete Empfehlungen zur Studienpla- nung und -durchführung umgesetzt werden - sowohl durch Early Dialogues als auch durch wiederholte, den Produktlebenszyklus begleitende Bewertungen reduziert werden.

- Ein europaweit harmonisierter Bewertungsprozess steigert durch einheitliche Methoden und Prozesse die Nachvollziehbarkeit der Assessments und das Vertrauen in die Qualität der Berichte, die so als evidenzbasierte Informationsgrundlage genutzt werden können. Zudem werden Kriterien, die für die Bewertung von Wirksamkeit und Sicherheit einer Technologie herangezogen werden, transparent dargestellt.

- Die Arbeitsteilung unter den HTA-Organisationen ermöglicht die Bewertung einer größeren Anzahl an Technologien mit den gleichen Ressourcen und damit die Sicherstellung evidenzbasierter Entscheidungen. Damit wird der zeitnahe Zugang zu tatsächlich wirksamen, sicheren und innovativen Technologien in den einzelnen Gesundheitssystemen erleichtert.

- Die Einbindung von Stakeholdern, insbesondere von PatientInnen (oder PatientenvertreterInnen), auf europäischer Ebene verbessert die standardisierte Berücksichtigung patientenrelevanter Endpunkte und Patientenpräferenzen.

- Auch wenn die europäischen Assessments keine Empfehlungen enthalten, regen sie den europäischen Diskurs zu Nutzen und Sicherheit neuer und etablierter Technologien an.

- Nicht zuletzt kann die Transparenz in der Zulassung und Bewertung von Medizinprodukten durch den europäischen Diskurs gesteigert werden.

\section{Ausblick auf die EUnetHTA JA3}

Die Wichtigkeit der grenzübergreifenden Zusammenarbeit im Bereich HTA zeigt sich in der Bereitschaft der Europäischen Kommission, eine weitere 4-jährige EUnetHTA JA (2016-2020) zu finanzieren. Über 70 europäische HTA-Organisationen nehmen an diesem Projekt teil, darunter große Organisationen wie das deutsche Institut für Qualität und Wirtschaftlichkeit im Gesundheitswesen (IQWiG) oder das britische National Institute for Health and Care Excellence (NICE). Das Ziel dieser JA3 ist die Festigung der internationalen Kollaboration auch nach Auslaufen der EU-Förderungen. Die Entwicklung von Strategien für ein permanentes und nachhaltiges HTA-Netzwerk ist substanzieller Bestandteil dieser JA3, um die Direktive 11/24/EU [1] auch nach 2020 zu erfüllen.

Die Europäische Kommission hat von Oktober 2016 bis Januar 2017 eine öffentliche Konsultation des „Inception Impact Assessment“ [18] durchgeführt, um die Ausgestaltung der Europäischen Zusammenarbeit im Bereich HTA auch nach EUnetHTA, also nach 2020, zu planen [19]. 5 verschiedene Modelle wurden aufgezeigt, beginnend bei einem rein freiwilligem Modell bis hin zu verpflichtender Zusammenarbeit anhand gesetzlicher Vorgaben. Noch sind keine Endergebnisse veröffentlicht, welches Modell präferiert wird; jedoch gaben laut ersten Informationen $92 \%$ der TeilnehmerInnen an, die Europäische HTA-Zusammenarbeit zu unterstützen [20].

Im Rahmen von 7 Arbeitspaketen werden während der JA3 Early Dialogues weitergeführt, Methoden zur Evidenzgenerierung nach Markteinführung entwickelt und gemeinsame Assessments zu Arzneimitteln und anderen Medizintechnologien erstellt. Die nationale Wiederverwendung, Qualität und Effizienz der gemeinsam er- 
stellten Assessments soll weiter gesteigert werden, um Redundanzen zu verhindern. Um sowohl der sich verändernden Evidenzlage im Laufe des Produktlebenszyklus und den Bedürfnissen der nationalen Entscheidungsträgerlnnen zu entsprechen, als auch eine europaweite Implementierung der EUnetHTA-Methoden zu unterstützen, werden 2 Formen von Assessments produziert: „Collaborative Assessments“ sollen durch schlankere Prozesse zeitnahe Informationen in einem frühen Stadium liefern. „Joint Assessments“ sind in einem späteren Stadium des Lebenszyklus geplant und sollen daher weiterhin mit breiter Stakeholder-Beteiligung und einem zentral koordinierten Priorisierungs- und Themenauswahl-Prozess erstellt werden.

Insbesondere die PatientInnenbeteiligung, die in der JA2 eine große Herausforderung darstellte, soll in der JA3 systematischer ablaufen: Patientlnnen können bspw. als Teil einer Fokusgruppe bereits im Scoping bei der Auswahl von relevanten Endpunkten einbezogen werden. Weitere Möglichkeiten betreffen die Beteiligung an Konsultationen von Projektprotokollen oder, für Ärztlnnen, die Mitwirkung als ExpertInnen. Relevante Informationen zu den laufenden Projekten sind auf der EUnetHTA Webseite (http:// www.eunethta.eu/) verfügbar.

Medizinprodukte umfassen ein breites Spektrum an Technologien, deren Bedeutung in den kommenden Jahrzehnten infolge des demografischen Wandels noch weiter zunehmen wird [13].

Um eine grenzüberschreitende Gesundheitsversorgung und die effiziente Zusammenarbeit der nationalen Gesundheitssysteme zu sichern, muss es auch in der Bewertung von Medizintechnologien mehr transnationale Kooperation zwischen HTA-Organisationen geben. Neben der gemeinsamen Bewertung sollte der Fokus daher auch auf einer flächendeckenden Implementierung gemeinsam entwickelter Methoden und Qualitätsstandards liegen; nur so können nachhaltige Strukturen europäischer Kooperation gebildet und das gegenseitige Vertrauen gefestigt werden. Durch eine vermehrt dezentrale Koordination der Berichterstellung innerhalb EUnetHTAs wird ein Multiplikatoreffekt erzielt: im Laufe der nächsten Jahre werden mehrere HTA-Organisationen zu „Activity Centers“ ausgebildet, die über die nötigen Projektmanagement-Skills verfügen, um internationale Assessments, erstellt mit EUnetHTA-Methoden, -Qualitätsstandards und -Werkzeugen, zu koordinieren.

Die nächsten Jahre werden zeigen, inwiefern bereits entwickelte Prozesse angepasst und neue Prozesse entwickelt werden müssen, um die nachhaltige Zusammenarbeit verwirklichen zu können. Gerade im Bereich der PatientInnenbeteiligung gilt es, neue Ansätze zu erproben und diese wichtige Stakeholdergruppe noch stärker in den Assessment-Prozess einzubeziehen.

\section{Schlussfolgerung}

Gelingt die Entwicklung und Umsetzung standardisierter Qualitätskriterien, kann die europäische Zusammenarbeit zu einem konkreten Nutzen für PatientInnen, ÄrztInnen, HTA-Organisationen und nationale EntscheidungsträgerInnen führen und zur Umsetzung der Direktive über die Ausübung der Patientenrechte in der grenzüberschreitenden Gesundheitsversorgung beitragen. Europäische Assessments können evidenzbasierte, nationale Kostenerstattungsentscheidungen zu Medizinprodukten unterstützen und so den Zugang zu sicheren Produkten gewährleisten, die einen tatsächlichen Patientennutzen aufweisen.

\section{Disclaimer}

Die in diesem Artikel vertretenen Ansichten reflektieren die Meinung der Autoren und nicht zwangsläufig die von EUnetHTA. EUnetHTA übernimmt keine Verantwortung für den Inhalt und dessen Weiterverwendung.

\section{Förderungen}

EUnetHTA wird von der Europäischen Kommission gefördert. Die alleinige Verantwortung für den Inhalt dieses Artikels liegt bei den Autorinnen. Die Europäische Kommission übernimmt keine Verantwortung für die Verwendung der darin enthaltenen Informationen.

Interessenkonflikt

Die Autorinnen geben an, dass kein Interessenkonflikt besteht.

\section{Literatur}

[1] European Parliament, Council of the European Union. Directive 2011/24/EU of the European Parliament and of the Council of 9 March 2011 on the application of patients' rights in cross-border healthcare. Official Journal of the European Union 2011; 54 (L 88): 45-65 Epub 04.04.2011

[2] Europäische Kommission. Grenzüberschreitende Pflege. Im Internet: http://ec.europa.eu/health/cross_border_care/policy/index_de.htm Stand: 31.05.2016

[3] Kristensen FB. Development of European HTA: from Vision to EUnetHTA. Michael Quarterly 2012; 9: 147-156

[4] European network for HTA. EUnetHTA Homepage. Im Internet: http:// www.eunethta.eu/ Stand: 20.04.2016

[5] European network for HTA. National Uptake. Im Internet: http://www. eunethta.eu/national-uptake Stand: 25.04.2016

[6] Council of the European Union. Council Directive 90/385/EEC of 20 June 1990 on the approximation of the laws of the Member States relating to active implantable medical devices. Official Journal of the European Union 1990; 33 (L 189): 17-36 Epub 20.07.1990

[7] Council of the European Union. Council Directive 93/42/EEC of 14 June 1993 concerning medical devices. Official Journal of the European Union 1993; 36 (L 169): 1-43 Epub 12.07.1993

[8] European Parliament, Council of the European Union. Directive 98/79/ EC of the European Parliament and of the Council of 27 October 1998 on in vitro diagnostic medical devices. Official Journal of the European Union 1998; 41 (L 331): 1-37 Epub 07.12.1998

[9] European Commission. Medical Devices: Regulatory Framework. Im Internet: http://ec.europa.eu/growth/sectors/medical-devices/ regulatory-framework/index_en.htm Stand: 06.06.2016

[10] Krüger L, Wild C. Evidence requirements for the authorization and reimbursement of high-risk medical devices in the USA, Europe, Australia and Canada: An analysis of seven high-risk medical devices. HTA-Projektbericht. Nr. 73 Wien: Ludwig Boltzmann Institut für Health Technology Assessment; 2013 
[11] European Commission. Notified Bodies Nando: Legislation. Im Internet: http://ec.europa.eu/growth/tools-databases/nando/index. $\mathrm{cfm}$ ?fuseaction $=$ directive . main Stand: 04.11.2016

[12] Schnell-Inderst P, Mayer J, Lauterberg J et al. Health Technology Assessment of medical devices: What is different? An overview of three European projects. Z Evid Fortbild Qual Gesundhwesen 2015; 109: 309-318

[13] Rat der Europäischen Kommission. Modernisierung der EU-Vorschriften für Medizinprodukte. Im Internet: http://www.consilium.europa. eu/de/policies/new-rules-medical-in-vitro-diagnostic-devices/ Stand: 25.06.2016

[14] European Commission. Revisions of Medical Device Directives. Im Internet: http://ec.europa.eu/growth/sectors/medical-devices/ regulatory-framework/revision/index_en.htm Stand:06.06.2016

[15] TÜV Süd International. Proposed EU Regulation for Medical Devices and Active Implantable Medical Devices. Im Internet: http://www. tuv-sud.com/industry/healthcare-medical-device/market-approvalcertification-for-medical-devices/ce-marking/mdr\#t ab_1452147199033922531592 Stand: 06.06.2016
[16] Reinhardt D, Wildner M. Inverkehrbringen von Arzneimitteln und Medizinprodukten in Deutschland: Evaluation der Verfahren und Schwachstellenanalyse. Das Gesundheitswesen 2016; 78: 844-852

[17] Fried A, Wild C. Beteiligung von BürgerInnen und PatientInnen in HTA Prozessen. Internationale Erfahrungen und Good Practice Beispiele. LBI-HTA Projektbericht Nr.: 86. Wien: Ludwig Boltzmann Institut für Health Technology Assessment; 2016

[18] Europäische Kommission. Inception Impact Assessment - Strengthening of the EU cooperation on Health Technology Assessment (HTA). 2016; Im Internet: http://ec.europa.eu/smart-regulation/roadmaps/ docs/2016_sante_144_health_technology_assessments_en.pdf Stand: 14.02.2017

[19] European Commission. Public consultation on strengthening EU cooperation on Health Technology Assessment (HTA). Im Internet: https://ec.europa.eu/health/technology_assessment/consultations/ cooperation_hta_en Stand: 14.02.2017

[20] Paun C. Majority supports EU cooperation on HTA, Commission says. POLITICO Pro. Im Internet: http://www.politico.eu/pro/majoritysupports-eu-cooperation-on-hta-commission-says/ Stand: 14.02.2017 